\title{
Mixed modulus and anomaly mediation in light of the muon $g-2$ anomaly
}

\author{
Kwang Sik Jeong, ${ }^{a}$ Junichiro Kawamura ${ }^{b, c}$ and Chan Beom Park ${ }^{b}$ \\ ${ }^{a}$ Department of Physics, Pusan National University, \\ Busan 46241, Korea \\ ${ }^{b}$ Center for Theoretical Physics of the Universe, Institute for Basic Science (IBS), \\ Daejeon 34126, Korea \\ ${ }^{c}$ Department of Physics, Keio University, \\ Yokohama 223-8522, Japan \\ E-mail: ksjeong@pusan.ac.kr, jkawa@ibs.re.kr, cbpark@ibs.re.kr
}

ABSTRACT: The new measurement of the anomalous magnetic moment of muon at the Fermilab Muon $g-2$ experiment has strengthened the significance of the discrepancy between the standard model prediction and the experimental observation from the BNL measurement. If new physics responsible for the muon $g-2$ anomaly is supersymmetric, one should consider how to obtain light electroweakinos and sleptons in a systematic way. The gauge coupling unification allows a robust prediction of the gaugino masses, indicating that the electroweakinos can be much lighter than the gluino if anomaly-mediated supersymmetry breaking is sizable. As naturally leading to mixed modulus-anomaly mediation, the KKLT scenario is of particular interest and is found capable of explaining the muon $g-2$ anomaly in the parameter region where the lightest ordinary supersymmetric particle is a bino-like neutralino or slepton.

Keywords: Supersymmetry Phenomenology

ArXiv EPrint: 2106.04238 


\section{Contents}

1 Introduction 1

2 Mixed modulus-anomaly mediation 3

3 The muon $g-2$ anomaly $\quad 7$

4 Collider signatures and LHC constraints $\quad 11$

$\begin{array}{lll}4.1 & \text { Stable neutralino LOSP } & 12\end{array}$

$\begin{array}{lll}4.2 & \text { Metastable slepton LOSP } & 14\end{array}$

$\begin{array}{lll}4.3 & \text { Unstable LOSP } & 15\end{array}$

$\begin{array}{lll}5 & \text { Summary } & 16\end{array}$

$\begin{array}{ll}\text { A Benchmark sparticle mass spectrum } & 17\end{array}$

\section{Introduction}

Since the experimental observation of the discrepancy at the Brookhaven National Laboratory (BNL) [1], the anomalous magnetic moment $g-2$ of muon has served as a long-standing puzzle of particle physics. Recently, the Fermilab Muon $g-2$ collaboration has announced the new measurement result [2], which has further strengthened the significance of the BNL result on the muon $g-2$. Comparing to the Standard Model (SM) prediction [3-23], the combined BNL and Fermilab result amounts be a $4.2 \sigma$ discrepancy. The deviation from the SM prediction is

$$
\Delta a_{\mu}=a_{\mu}^{\mathrm{exp}}-a_{\mu}^{\mathrm{SM}}=(25.1 \pm 5.9) \times 10^{-10},
$$

where $a_{\mu} \equiv\left(g_{\mu}-2\right) / 2$. Although the recent lattice calculation for the hadronic vacuum polarization contribution to the muon $g-2$ has turned out to be in accord with the measured value $[24],{ }^{1}$ we take this opportunity to examine the possibility of new physics accounting for the muon $g-2$ anomaly.

Among various possible models that can explain the muon $g-2$ anomaly, we consider the supersymmetric (SUSY) model as it is one of the most promising candidates. Ever since the announcement of the Fermilab result has come out, the SUSY interpretations for the muon $g-2$ anomaly have already been revisited or renewed in many works [29-51]. In the Minimal Supersymmetric Standard Model (MSSM), the contributions to the muon

\footnotetext{
${ }^{1}$ In refs. [25-28], it has been claimed that shifting the hadronic vacuum polarization value of the SM to match the measured value of the muon $g-2$ would result in tension with the global fit to electroweak precision data.
} 
$g-2$ can be generated by bino, winos, Higgsinos, smuons, and sneutrino. For sparticle masses of order $M_{\mathrm{SUSY}}$, the leading SUSY contributions have the generic behavior of

$$
\Delta a_{\mu}^{\mathrm{SUSY}} \propto \frac{m_{\mu}^{2} \mu M_{a}}{M_{\mathrm{SUSY}}^{4}} \tan \beta
$$

where $\mu$ is the Higgsino mass, $M_{a}$ is the gaugino mass, and $\tan \beta$ is the ratio of the Higgs vacuum expectation values [52]. Their relative contributions can differ by the sparticle mass spectrum. For example, if the Higgsinos are very heavy, only the bino-smuon loop contribution can become sizable. Whereas the sfermion masses are highly model dependent, the gaugino masses show a robust pattern owing to the gauge coupling unification at the grand unified theory (GUT) scale, $M_{\text {GUT }}$ [53]. For instance, in gravity mediation with universal gaugino masses at $M_{\mathrm{GUT}}$ [54] or in gauge mediation with messengers forming a GUT multiplet [55-57], the ratios of low energy gaugino masses read

$$
M_{1}: M_{2}: M_{3} \simeq 1: 2: 6
$$

at the $\mathrm{TeV}$ scale, regardless of the details of the model. Anomaly mediation [58, 59], which always exists in supergravity, modifies the above gaugino mass relation depending on its relative strength, and may make the wino and/or bino much lighter than the gluino as is required to explain the muon $g-2$ anomaly. A natural framework for sizable anomaly mediation is provided by the KKLT string compactification [60]. A remarkable feature of the KKLT moduli stabilization is that the parameters of SUSY breaking are, in principle, controlled by discrete numbers, such as the winding number of D-branes, the number of fluxes that generate moduli potential, and so on.

In this article, we point out that mixed modulus-anomaly mediation [61-65], which is realized in the KKLT setup, can accommodate light electroweakinos (EWinos) and sleptons to explain the muon $g-2$ anomaly and heavy colored sparticles to evade the lower limits from the LHC. To obtain the suitable sparticle mass spectra for the muon $g-2$, we need to consider a generalized KKLT setup beyond the minimal one, as described in section 2. By imposing various conditions such as the Higgs boson properties and the vacuum stability of the scalar potential, we perform numerical analysis in the parameter space to check the feasibility of the model. We present our analysis result in section 3. Although our result is mostly safe from the lower limits set by the search results on colored sparticles at the LHC, the bounds from the searches for the chargino-neutralino and the slepton pair productions may exclude the parameter points of light sleptons and gauginos. We also find that, in a large part of the parameter space for the muon $g-2$, a slepton becomes lighter than the lightest neutralino. In this case, we should consider alternative scenarios such as a Peccei-Quinn (PQ) symmetric extension where the axino is the lightest sparticle or Rparity violating (RPV) interactions to make the lightest ordinary SUSY particle (LOSP), the lightest sparticle among the MSSM sparticles, unstable. The LHC limits and the phenomenological scenarios with light sleptons are discussed in section 4 . We summarize our study in the last section. For reference, we list the benchmark sparticle mass spectra in appendix A. 


\section{Mixed modulus-anomaly mediation}

The sparticle mass spectrum crucially depends on how SUSY breaking in a hidden sector is transmitted to the visible sector. To be consistent with the experimental constraints, SUSY breaking mediation should preserve flavor and CP symmetry with good accuracy unless it makes the sparticles very heavy above $100 \mathrm{TeV}$. Indeed, many mediation schemes such as gravity mediation, dilaton/moduli mediation, gaugino mediation, gauge mediation, anomaly mediation, and their mixtures conserve flavor and CP symmetry and lead to various patterns of sparticle spectra. Among the sparticles, the gauginos are known to have a robust pattern of masses under the condition of gauge coupling unification [53]. The gaugino masses in mixed modulus-anomaly mediation are written as

$$
M_{a}=M_{0}\left(1+\frac{b_{a} g_{\mathrm{GUT}}^{2}}{4} \alpha\right)
$$

at the scale just below $M_{\mathrm{GUT}}$, where the gauge coupling constants have the common value, $g_{a}^{2}\left(M_{\mathrm{GUT}}\right)=g_{\mathrm{GUT}}^{2}$. Here, $b_{a}=(33 / 5,1,-3)$ are the coefficients of the one-loop beta functions at $\mathrm{TeV}$, and the $\alpha$ parameter represents the relative strength of anomaly mediation:

$$
\alpha \equiv \frac{m_{3 / 2}}{4 \pi^{2} M_{0}},
$$

with $m_{3 / 2}$ being the gravitino mass. Note that anomaly mediation is a model-independent supergravity effect proportional to the gravitino mass, but it alone suffers from the tachyonic slepton problem. Because the combination $M_{a} / g_{a}^{2}$ is renormalization group (RG) invariant at one loop, the gaugino masses at the $\mathrm{TeV}$ scale are found to approximately obey the following relation:

$$
M_{1}: M_{2}: M_{3} \simeq(1+0.83 \alpha):(2+0.25 \alpha):(6-2.25 \alpha),
$$

where we have taken $g_{\mathrm{GUT}}^{2}=0.5$ and used the ratios of the gauge couplings $g_{1}^{2}: g_{2}^{2}: g_{3}^{2} \simeq$ $1: 2: 6$ at the $\mathrm{TeV}$ scale.

As the first explicit realization of a de Sitter (dS) vacuum with all string moduli stabilized, the KKLT mechanism [60] provides an interesting framework to realize mixed modulus-anomaly mediation with $\alpha$ of order unity. In the minimal KKLT setup, $\alpha$ is a positive rational number $[61,62]$. In the literature, the phenomenology of positive $\alpha$ has been studied intensively particularly to resolve the fine-tuning problem to realize the electroweak (EW) symmetry breaking scale [66-72]. However, the muon $g-2$ anomaly is hardly explained in the cases with positive $\alpha$ due to relatively heavy winos and bino unless the other contribution exists [68, 73]. One can generalize the KKLT setup to obtain a negative $\alpha$ to get a desired mass hierarchy in the gaugino spectrum. For a concrete discussion, let us consider a model where the effective moduli superpotential is given by ${ }^{2}$

$$
W=A_{0} e^{-4 \pi^{2} \ell_{0} S_{0}}-A_{1} e^{-4 \pi^{2}\left(k_{1} T+\ell_{1} S_{0}\right)},
$$

\footnotetext{
${ }^{2}$ We take the reduced Planck mass unit, $M_{P l}=1$, unless stated otherwise.
} 
with $A_{0}$ and $A_{1}$ being constants of order unity, and the visible sector gauge kinetic function is written as

$$
f_{a}=k T+\ell S_{0}
$$

after integrating out the heavy dilation $S$ and complex structure moduli fixed by fluxes at $S=S_{0}$. Here, $\ell_{0} / \ell, \ell_{1} / \ell$, and $k_{1} / k$ are rational numbers determined by topological or group theoretical data of the underlying string compactification, as can be dictated from the periodicities of $\operatorname{Im}(T)$ and $\operatorname{Im}(S)$. A supersymmetric minimum is developed by the above superpotential and is lifted to a dS vacuum by adding a SUSY breaking uplifting potential that originates from a brane-localized source located at the IR end of the warped throat. The uplifting potential is given by

$$
V_{\text {lift }}=\frac{P e^{2 K_{0} / 3}}{\left(T+T^{*}\right)^{n_{P}}}
$$

for a rational number $n_{P} . \quad P$ is a positive constant fixed by the condition of vanishing cosmological constant. Here, the modulus Kähler potential generally reads

$$
K_{0}=-n_{0} \ln \left(T+T^{*}\right),
$$

for a positive rational number $n_{0}$. An extra-dimensional interpretation of the uplifting procedure is possible for $n_{P} \geq 0$, because otherwise the uplifting sector couples more strongly for a larger value of $T$ [74]. In the above model, one finds $\alpha$ to be [68]

$$
\alpha \equiv \frac{m_{3 / 2}}{4 \pi^{2} M_{0}} \simeq \frac{2 k_{1}}{k}\left(1+\frac{3 n_{P}}{2 n_{0}}\right)^{-1}
$$

from the observations that gauge coupling unification requires $g_{\mathrm{GUT}}^{-2}=\operatorname{Re}\left(f_{a}\right) \simeq 2$, and that $T$ is stabilized at $k_{1} T \simeq\left(\ell_{0}-\ell_{1}\right) S_{0}$ with $S_{0}$ written in terms of the gravitino mass as $4 \pi^{2} \ell_{0} \operatorname{Re}\left(S_{0}\right) \simeq \ln \left(M_{P l} / m_{3 / 2}\right)$. Note that $M_{0}$ is given by $M_{0}=F^{T} \partial_{T} \ln \operatorname{Re}\left(f_{a}\right)$ with $F^{T}$ being the modulus $F$-term. A negative $\alpha$ is therefore obtained if either $k_{1}$ or $k$ is negative. See, for example, ref. [75] for more discussion on the case with $k_{1}<0$ and $k>0$.

Let us continue to examine the sfermion masses, which generally possess a stronger model dependence compared to the gaugino masses. In the mixed modulus-anomaly mediation under consideration, the sfermion masses are determined by the modulus dependence of the matter Kähler potential:

$$
K=-n_{0} \ln \left(T+T^{*}\right)+\frac{\Phi_{i}^{*} \Phi_{i}}{\left(T+T^{*}\right)^{n_{i}}} .
$$

We have taken into account a simple case where the matter Kähler metric is not affected by the involved dilaton-modulus mixing. Here, the modular weight $n_{i}$ is a rational number of order unity fixed by the location of the matter in extra dimensions. The mixed mediation in the KKLT preserves CP and flavor symmetries respectively due to the axionic shift symmetry associated with $T$ and flavor-universal modular weights. The pure modulusmediated (MM) trilinear $A$-parameters and soft scalar masses are found to be

$$
\begin{aligned}
\left.A_{i j k}\right|_{\mathrm{MM}} & =-\left(a_{i}+a_{j}+a_{k}\right) M_{0}, \\
\left.m_{i}^{2}\right|_{\mathrm{MM}} & =c_{i} M_{0}^{2},
\end{aligned}
$$


at $M_{\mathrm{GUT}}$, where $a_{i}$ and $c_{i}$ are given by

$$
\begin{aligned}
a_{i} & =\left(\frac{n_{0}}{3}-n_{i}\right)\left(1+\frac{k_{1}}{k} \frac{\ell}{\ell_{0}-\ell_{1}}\right), \\
c_{i} & =\left(1+\frac{k_{1}}{k} \frac{\ell}{\ell_{0}-\ell_{1}}\right) a_{i} .
\end{aligned}
$$

Note that $a_{i}$ and $c_{i}$ are rational numbers of either sign depending on the choice of the associated discrete numbers.

It is worth noting that an anomalous $\mathrm{U}(1)_{A}$ gauge symmetry allows the $\alpha$ parameter and the effective modular weights to have various values in a much wider range if $T$ transforms non-linearly to implement the Green-Schwarz (GS) anomaly cancellation mechanism [76]. Let us consider a simple case where the modulus-induced Fayet-Iliopoulos term is canceled by a single $\mathrm{U}(1)_{A}$ charged but $\mathrm{SM}$ singlet matter field $X$. Integrating out the heavy $\mathrm{U}(1)_{A}$ gauge superfield, whose longitudinal component comes mostly from $X$, one can construct the low energy effective theory of a light modulus $T$, which is mainly the GS modulus, and light matter fields. The Kähler potential reads [77]

$$
K_{\mathrm{eff}}=-n_{0} \ln \left(T+T^{*}\right)+\frac{\Phi_{i}^{*} \Phi_{i}}{\left(T+T^{*}\right)^{n_{i}^{\mathrm{eff}}}},
$$

with the effective modular weight given by

$$
n_{i}^{\mathrm{eff}} \simeq n_{i}+\left(1-n_{X}\right) \frac{q_{i}}{q_{X}},
$$

where $n_{\alpha}$ and $q_{\alpha}$ are the modular weight and $\mathrm{U}(1)_{A}$ charge of the corresponding field, respectively. From the fact that the superpotential is a holomorphic function of the $\mathrm{U}(1)_{A}$ invariant combination of the GS modulus and $X$, the effective superpotential is found to be

$$
W_{\text {eff }}=A_{0} e^{-4 \pi^{2} \ell_{0} S_{0}}-A_{1} e^{-4 \pi^{2}\left(k_{1} T+k_{H}+\ell_{1} S_{0}\right)} .
$$

for the constants $A_{0}$ and $A_{1}$ of order unity. Here, $k_{H}$ is a constant of order unity fixed by the $\mathrm{U}(1)_{A}$ invariance. It is then straightforward to see that $\alpha$ is given by

$$
\alpha \simeq\left(1-\frac{4 \pi^{2}}{\ln \left(M_{P l} / m_{3 / 2}\right)} k_{H}\right) \times \frac{2 k_{1}}{k}\left(1+\frac{3 n_{P}}{2 n_{0}}\right)^{-1} .
$$

The above shows that a positive $k_{H}$ can flip the sign of $\alpha$, implying that $\mathrm{U}(1)_{A}$ not only enlarges the possible range of modular weights, but also makes it possible to achieve a negative $\alpha$ in the moduli stabilization with $k k_{1}>0$. Meanwhile, the holomorphic Yukawa coupling changes as

$$
\lambda_{i j k} \rightarrow \lambda_{i j k} \epsilon^{-\left(q_{i}+q_{j}+q_{k}\right) / q_{X}},
$$

because it arises from the superpotential, $X^{n_{i j k}} \Phi_{i} \Phi_{j} \Phi_{k}$, where $n_{i j k}=-\left(q_{i}+q_{j}+q_{k}\right) / q_{X}$ should be a non-negative integer. $\epsilon \sim 0.1$ represents the VEV of $X$ relative to $M_{P l}$. It is clear that a large Yukawa coupling $y_{i j k}$ of order unity apparently constrains the $\mathrm{U}(1)_{A}$ charges to be $n_{i j k}=0$ or $1 .^{3}$

\footnotetext{
${ }^{3}$ In the case where $n_{X}=1$, one can assign flavor-dependent $\mathrm{U}(1)_{A}$ charges, for which $\mathrm{U}(1)_{A}$ can account for the hierarchical Yukawa couplings via the Froggatt-Nielsen mechanism [78].
} 


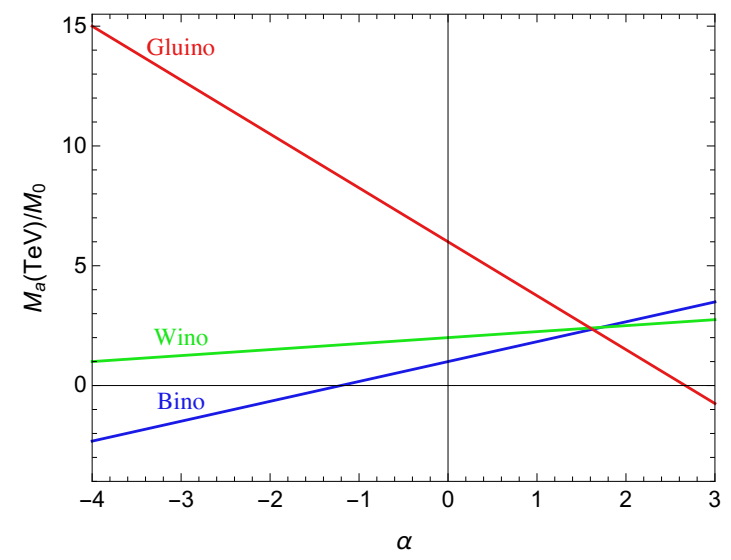

Figure 1. Gaugino masses at $\mathrm{TeV}$ as functions of the $\alpha$ parameter. The masses have been normalized by $M_{0}$, which is the pure modulus-mediated contribution at $M_{\mathrm{GUT}}$. The red, green, and blue colored lines correspond to the gluino, wino, and bino masses, respectively.

To summarize, the generalized KKLT setup leads to mixed modulus-anomaly mediation where the sparticle masses are determined by three types of dimensionless parameters:

$$
\alpha, \quad a_{i}, \quad c_{i}
$$

with $c_{i} \propto a_{i}$. The parameters can take various values of order unity with either sign, leading to a variety of sparticle mass spectra. The overall size of sparticle masses is fixed by $M_{0}$. While achieving gauge coupling unification, the gauginos show a robust mass relation given by eq. (2.3) at the $\mathrm{TeV}$ scale, and interestingly, they can have a large mass hierarchy for a negative $\alpha$. In figure 1, we display the gaugino masses as functions of the $\alpha$ parameter, including negative values. It shows that gluinos can become much heavier than the others for a largely negative $\alpha$. As discussed in the next sections, this feature is essential for evading the collider bounds while explaining the muon $g-2$ anomaly with light EWinos. Meanwhile, the sfermion soft parameters just below the unification scale are given by ${ }^{4}$

$$
\begin{aligned}
& \frac{A_{i j k}}{M_{0}}=-\left(a_{i}+a_{j}+a_{k}\right)-\frac{\alpha}{4}\left(\gamma_{i}+\gamma_{j}+\gamma_{k}\right), \\
& \frac{m_{i}^{2}}{M_{0}^{2}}=c_{i}+\left(\sum_{j k}\left(a_{i}+a_{j}+a_{k}\right)\left|y_{i j k}\right|^{2}-4 k \sum_{a} g_{a}^{2} C_{a}\left(\Phi_{i}\right)\right) \frac{\alpha}{4}+\dot{\gamma}_{i}\left(\frac{\alpha}{4}\right)^{2},
\end{aligned}
$$

for $f_{a}=k T+\Delta f_{a}$ with $\Delta f_{a}$ depending on other moduli of the model. The anomalous dimension $\gamma_{i}$ is given by

$$
16 \pi^{2} \gamma_{i}=\frac{1}{2} y^{i m n} y_{i m n}-2 g_{a}^{2} C_{a}\left(\Phi_{i}\right)
$$

\footnotetext{
${ }^{4}$ We employ the convention in the SUSY Les Houches Accord (SLHA) format [79] with $M_{3}$ being positive for $\alpha \lesssim 2.5$. For example, the parameters in ref. [68] can be obtained by redefining $M_{a} \rightarrow-M_{a}, A_{i j k} \rightarrow$ $-A_{i j k}$ and $\mu \rightarrow-\mu$. It corresponds to the field redefinitions: $\lambda_{a} \rightarrow i \lambda_{a}, \psi_{i} \rightarrow i \psi_{i}$ and $\phi_{i} \rightarrow-\phi_{i}$, where $\lambda_{a}, \psi_{i}$ and $\phi_{i}$ are gauginos, Weyl fermions, and scalars, respectively. The other coupling constants are unchanged under the field redefinition. The signs of the gaugino mass terms in the SLHA format are opposite from those in ref. [68]. Here, $A_{i j k}$ is a trilinear coupling divided by a Yukawa coupling constant $y_{i j k}$.
} 
and $\dot{\gamma}=8 \pi^{2} d \gamma_{i} / d \ln Q$ with $Q$ being the RG scale. $C_{a}\left(\Phi_{i}\right)$ is the quadratic Casimir invariant of $\Phi_{i}$.

\section{The muon $g-2$ anomaly}

We are now in a position to examine the possibility of explaining the muon $g-2$ anomaly in mixed modulus-anomaly mediation realized in the generalized KKLT setup. Depending on sparticle mass spectrum, various different SUSY contributions can enhance (or reduce) the muon $g-2$. For a recent review on the SUSY contributions in light of the muon $g-2$ anomaly, we refer the reader to ref. [43] and the references therein. In the MSSM, the most important contributions to the muon $g-2$ arise from the Higgsino-wino-smuon (HWL) and bino-smuon (BLR) loop diagrams, which are given respectively by

$$
\begin{aligned}
\Delta a_{\mu}^{\mathrm{HWL}} & =\frac{g_{2}^{2}}{8 \pi^{2}} \frac{m_{\mu}^{2} M_{2}}{m_{\tilde{\mu}_{L}}^{4}} \mu \tan \beta\left[F_{a}\left(\frac{M_{2}^{2}}{m_{\tilde{\mu}_{L}}^{2}}, \frac{\mu^{2}}{m_{\tilde{\mu}_{L}}^{2}}\right)-\frac{1}{2} F_{b}\left(\frac{M_{2}^{2}}{m_{\tilde{\mu}_{L}}^{2}}, \frac{\mu^{2}}{m_{\tilde{\mu}_{L}}^{2}}\right)\right], \\
\Delta a_{\mu}^{\mathrm{BLR}} & =\frac{g_{1}^{2}}{8 \pi^{2}} \frac{m_{\mu}^{2}}{M_{1}^{3}} \mu \tan \beta F_{b}\left(\frac{m_{\tilde{\mu}_{L}}^{2}}{M_{1}^{2}}, \frac{m_{\tilde{\mu}_{R}}^{2}}{M_{1}^{2}}\right) .
\end{aligned}
$$

The expressions for the loop functions $F_{a}$ and $F_{b}$ can be found in e.g. ref. [43]. As will be discussed shortly, the Higgsinos, whose mass is tied to the up-type Higgs soft mass under the condition of EW symmetry breaking, are relatively heavy compared to other particles relevant to the muon $g-2$. In such a case, the SUSY contribution to the muon $g-2$ mostly comes from the BLR one because the $\Delta a_{\mu}^{\mathrm{HWL}}$ is suppressed by large $\mu$. For the same reason, the other loop effects involving the Higgsinos are subdominant. Assuming that one of the smuons is significantly lighter than the other one, the SUSY contributions to the muon $g-2$ are approximately given by

$$
\begin{aligned}
\Delta a_{\mu}^{\mathrm{SUSY}} & \approx \frac{g_{1}^{2}}{8 \pi^{2}} \frac{m_{\mu}^{2} \mu}{m_{\tilde{\ell}_{+}}^{2} M_{1}} \tan \beta \times F_{\mathrm{B}}\left(\frac{m_{\tilde{\ell}_{-}}^{2}}{M_{1}^{2}}\right) \\
& \simeq 2.5 \times 10^{-9}\left(\frac{500 \mathrm{GeV}}{m_{\tilde{\ell}_{+}}}\right)^{2}\left(\frac{250 \mathrm{GeV}}{M_{1}}\right)\left(\frac{\mu}{2 \mathrm{TeV}}\right)\left(\frac{\tan \beta}{25}\right)\left(\frac{F_{\mathrm{B}}\left(m_{\tilde{\ell}_{-}}^{2} / M_{1}^{2}\right)}{1 / 6}\right)
\end{aligned}
$$

where

$$
F_{B}(x)=\frac{-1+x^{2}-2 x \ln x}{2(x-1)^{3}}
$$

is the loop function. Here, $m_{\tilde{\ell}_{-}\left(\tilde{\ell}_{+}\right)}$is the lighter (heavier) smuon mass. The expression is valid as long as the SUSY contributions to the muon $g-2$ are dominated by the BLR contribution and the bino is much lighter than the heavier smuon. It shows that the sign of the Higgsino mass $\mu$ and the bino mass $M_{1}$ must be matched to have a positive contribution to $\Delta a_{\mu}^{\mathrm{SUSY}}$.

As we have seen in section 2, the sparticle mass spectrum in mixed modulus-anomaly mediation is governed by the three types of dimensionless parameters, $\alpha, a_{i}, c_{i}$, as well as 
$M_{0}$. Here we fix the $M_{0}$ value by requiring that the lightest CP-even Higgs boson, whose properties approach to those of the SM Higgs boson in the decoupling limit, should have mass, $m_{h} \simeq 125 \mathrm{GeV}$, to be compatible with the observation [74]. For the sake of simplicity, we take

$$
a_{i}=c_{i},
$$

which corresponds to the case where the visible gauge kinetic function depends only on $T$, i.e. the case with $\ell=0$ in (2.5), as in the minimal KKLT [62]. Furthermore, motivated by the flavor constraints and the unification of gauge couplings, we assume that the modular weights respect the flavor universality and follow the SU(5) GUT relations for quarks and leptons,

$$
c_{5} \equiv c_{L}=c_{D}, \quad c_{10} \equiv c_{Q}=c_{U}=c_{E} .
$$

In our analysis, therefore, the input parameters of the model are given as follows:

$$
\alpha, \quad \tan \beta, \quad \operatorname{sgn}(\mu), \quad c_{5}, \quad c_{10}, \quad c_{H_{u}}, \quad c_{H_{d}} .
$$

Note that the size of $\mu$ is determined by the condition of EW symmetry breaking, and we take both signs of $\mu$ because $M_{1}$ can have either sign depending on the value of $\alpha$. At low energy scales, the mass splittings of squarks and sleptons are induced by the RG effects involved with the gauginos, and anomaly-mediated contributions.

To investigate the parameter space of mixed modulus-anomaly mediation, we have added the boundary conditions of mixed mediation to the SOFTSUSY program [80] and calculated the sparticle and Higgs mass spectra using the program. We require that the SM-like Higgs boson mass calculated with SOFTSUSY is within $125.10 \pm 0.01 \mathrm{GeV}$. Then, for each parameter point, we obtain the SUSY contributions to $\Delta a_{\mu}$ by using GM2Calc, which can compute the muon $g-2$ up to two-loop corrections [81].

Before looking into our analysis results, we should consider some theoretical constraints. The sleptons are required to be light to explain the muon $g-2$ anomaly, and the lightest stau can become tachyonic due to the large values of $|\mu| \gtrsim 2 \mathrm{TeV}$ leading to a large mixing angle. In our analysis, we discarded the parameter points with any tachyonic sfermion, including sleptons, flagged by SOFTSUSY. Furthermore, we should avoid the possibility of having a dangerous charge-breaking minimum in the scalar potential deeper than the EW vacuum. As it is not checked by SOFTSUSY, we impose the vacuum stability condition given in ref. [82]: for

$$
\begin{aligned}
\tilde{\eta}_{\ell}= & \left|m_{\tilde{\ell}_{L R}}^{2}\right| \times\left[101 \mathrm{GeV}\left(\sqrt{m_{\tilde{\ell}_{L}} m_{\tilde{\ell}_{R}}}+m_{\tilde{\ell}_{L}}+1.03 m_{\tilde{\ell}_{R}}\right)\right. \\
& \left.-2.27 \times 10^{4} \mathrm{GeV}^{2}+\frac{2.97 \times 10^{6} \mathrm{GeV}^{3}}{m_{\tilde{\ell}_{L}}+m_{\tilde{\ell}_{R}}}-1.14 \times 10^{8} \mathrm{GeV}^{4}\left(\frac{1}{m_{\tilde{\ell}_{L}}^{2}}+\frac{0.983}{m_{\tilde{\ell}_{R}}^{2}}\right)\right]^{-1},
\end{aligned}
$$

we require

$$
\tilde{\eta}_{\ell}<\eta_{\ell},
$$

where $\ell=\mu, \tau$. In eq. (3.8), $m_{\tilde{\ell}_{L}}^{2}, m_{\tilde{\ell}_{R}}^{2}$, and $m_{\tilde{\ell}_{L R}}^{2}$ are the diagonal element of lefthanded smuon, that of right-handed smuon, and the off-diagonal element in the smuon 

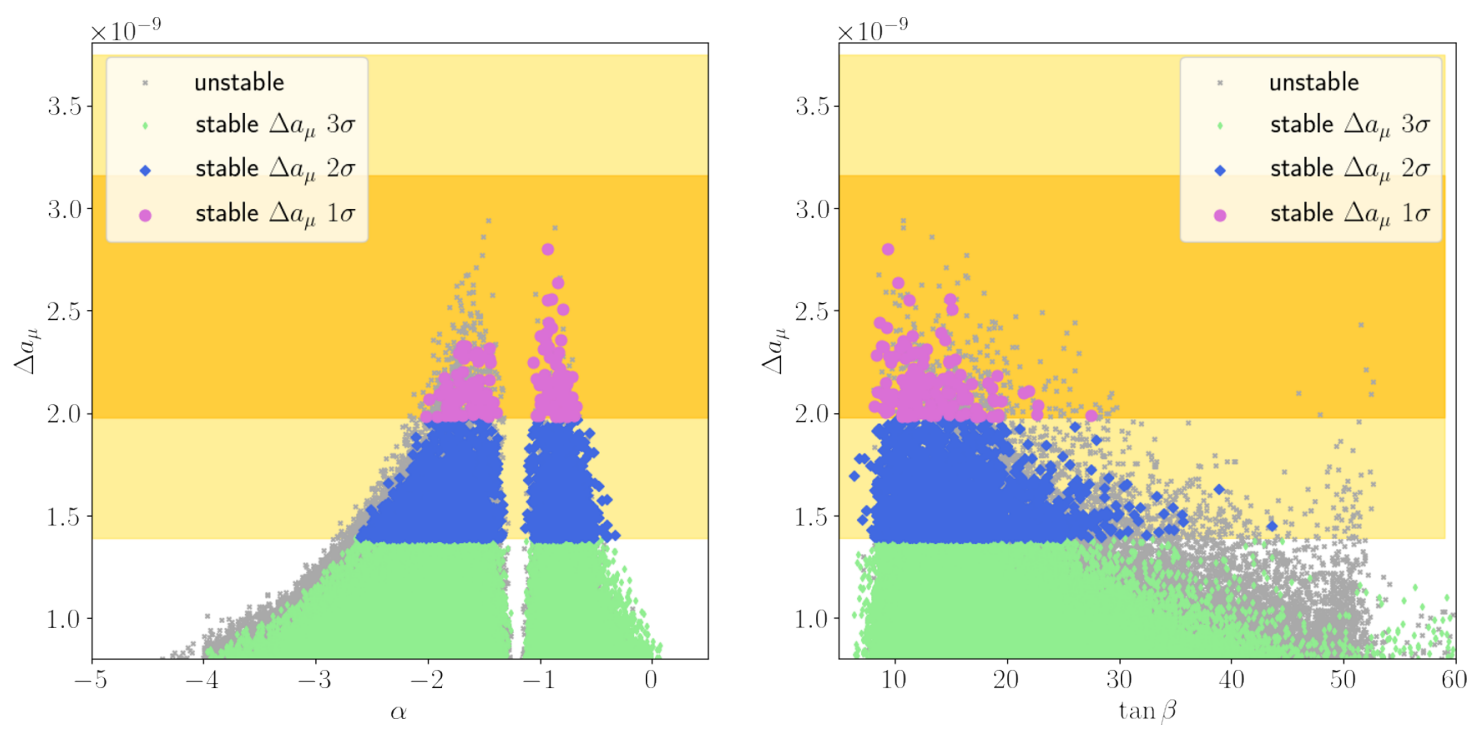

Figure 2. Parameter points of $\alpha$ (left) and $\tan \beta$ (right) compatible with the muon $g-2$ anomaly. The orange and yellow bands correspond respectively to the $1 \sigma$ and $2 \sigma$ ranges of the measured $\Delta a_{\mu}$ value, and accordingly, the parameters points are colored in magenta $(1 \sigma)$, blue $(2 \sigma)$, and green $(3 \sigma)$. Points colored in gray violate the vacuum stability condition.
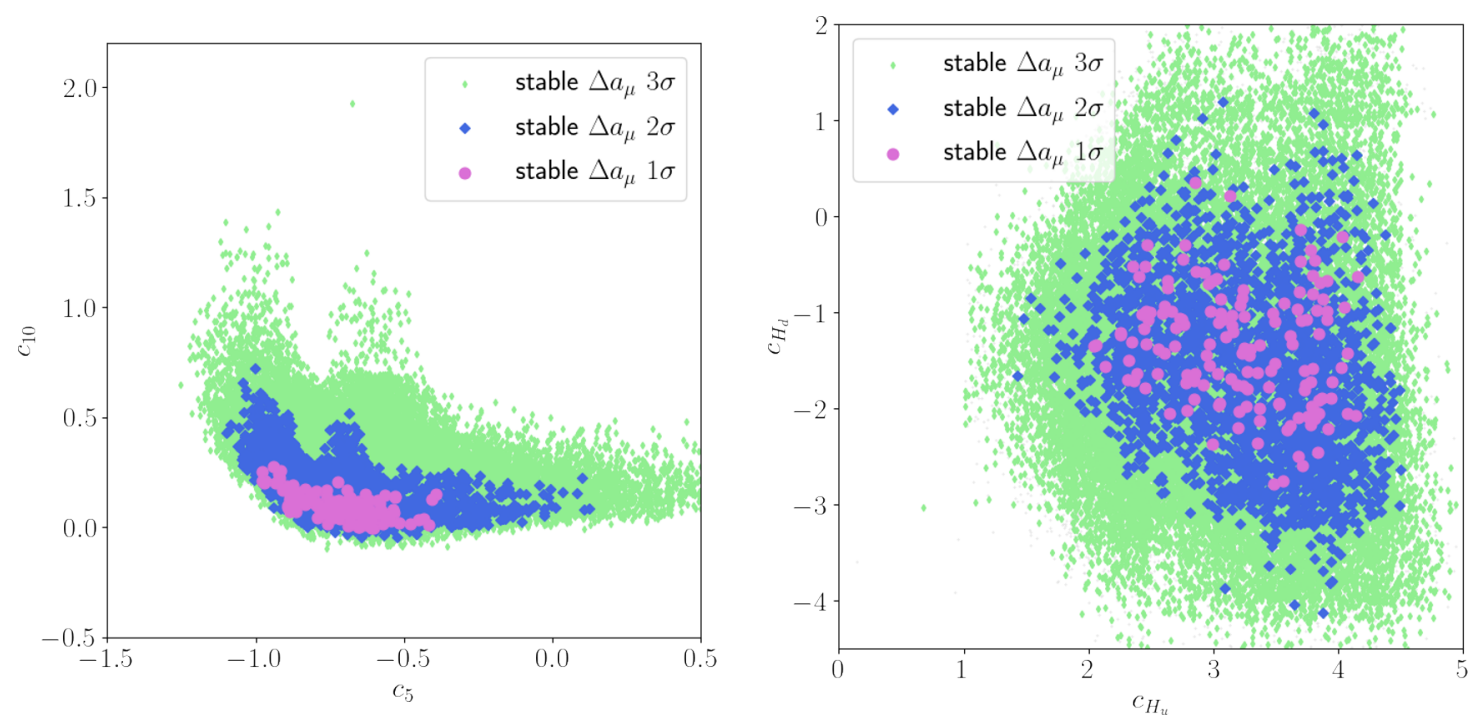

Figure 3. Parameter points in the space of $\left(c_{5}, c_{10}\right)$ (left) and $\left(c_{H_{u}}, c_{H_{d}}\right)$ (right). The points colored in magenta, blue, and green can explain the muon $g-2$ anomaly within $1 \sigma, 2 \sigma$, and $3 \sigma$, respectively.

mass squared matrix, respectively. Ignoring the small $\tan \beta$ dependence, we take $\eta_{\tau}\left(\eta_{\mu}\right)$ $=0.94(0.88)$ [83].

We now discuss our analysis results. In figure 2, we display the parameter points of $\alpha$ and $\tan \beta$, which can explain the muon $g-2$ anomaly within $3 \sigma$, on the left and right panels, respectively. The results show that the muon $g-2$ prefers the negative values of $\alpha$, where bino is light: $M_{1} \simeq 100-200 \mathrm{GeV}$. In particular, the $\Delta a_{\mu}$ value is the most sizable 


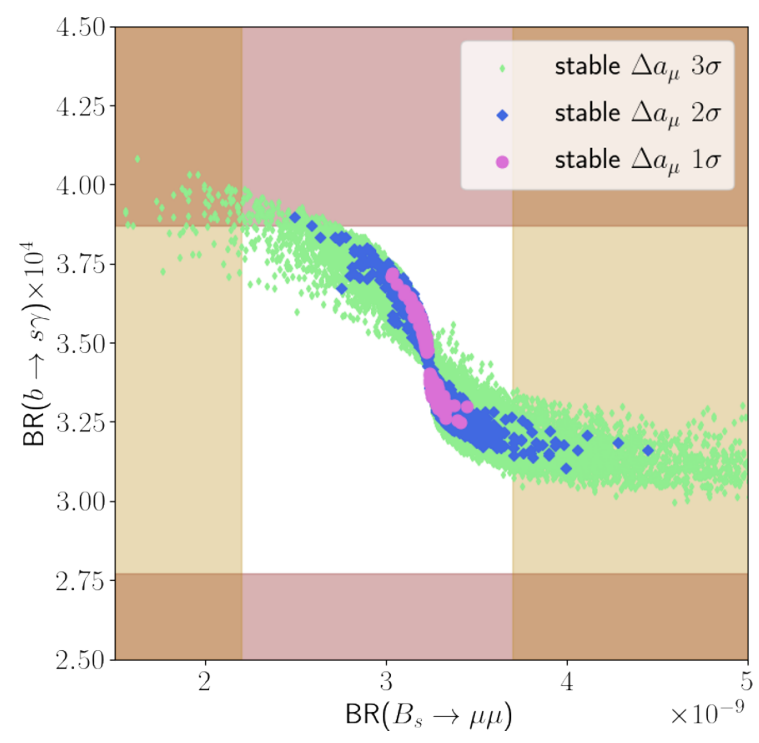

Figure 4. Scattering plot on $\left(\mathrm{BR}\left(B_{s} \rightarrow \mu \mu\right), \mathrm{BR}(b \rightarrow s \gamma)\right)$. The color coding of the points is the same as in the previous figures. The colored regions deviate from the experimental central values by more than $2 \sigma$.

when $\alpha \simeq-0.8$ or -1.6 . In the former case, $M_{1}$ is positive, whereas in the latter case, it is negative as can be seen in figure 1. There is a gap between the two cases, where $M_{1}$ becomes very small. In the gap, either the $S=\operatorname{Tr}\left[Y_{i} m_{\phi_{i}}^{2}\right]$ or the $A_{\tau}$ terms can drive the right-handed stau tachyonic in the RG running. The right panel of figure 2 shows that a wide range of $\tan \beta$ can be compatible with the measured $\Delta a_{\mu}$. However, when $\tan \beta$ is large ( $\gtrsim 30)$, a deeper charge-breaking vacuum can be induced even if $\Delta a_{\mu}$ will be enhanced by $\tan \beta$ as shown in eq. (3.3).

The SUSY contribution to the muon $g-2$ in mirage mediation has been studied in ref. [84]. Compared to the previous study, we find that a larger mass hierarchy between the wino/bino and the gluino is required in order to enhance $\Delta a_{\mu}$ while avoiding the experimental constraints, which is for $\alpha$ between about -3 and -0.5 , as can be seen in figure 2. Here, the constraints include the lower bound on the gluino mass from the LHC searches and the mass of the SM-like Higgs boson. Our analysis shows that the gluino has mass, $m_{\tilde{g}} \gtrsim 2.5 \mathrm{TeV}$, in the parameter region compatible with the muon $g-2$ anomaly. Another consequence of $\alpha$ in the indicated region is that the heavy gluinos drive the uptype Higgs soft mass squared, $m_{H_{u}}^{2}$, to negative and large in magnitude via RG evolution. This implies heavy Higgsinos because the EW symmetry breaking requires

$$
|\mu|^{2} \approx-m_{H_{u}}^{2}-\frac{1}{2} m_{Z}^{2}
$$

for moderate to large $\tan \beta$. It turns out that $|\mu| \gtrsim 2 \mathrm{TeV}$ in the parameter region for the muon $g-2$. Consequently, for $\alpha$ in the indicated region, the SUSY contributions are dominated by the BLR loop diagram, which is approximately proportional to the Higgsino mass. The BLR contribution can be enhanced further in the presence of light smuons below 
$1 \mathrm{TeV}$. We show the sparticle masses in the next section, and benchmark points are given in appendix A.

As described in section 2, the modular weights $c_{i}$ are rational numbers in the KKLT setup. In our analysis, we have taken them to be real positive or negative numbers to find the viable ranges of $c_{i}$ that can explain the muon $g-2$ anomaly. The result of our parameter scan is shown in figure 3 . We find that the favored regions are $0 \lesssim c_{10} \lesssim 0.5$, and $-1 \lesssim c_{5} \lesssim 0,2 \lesssim c_{H_{u}} \lesssim 4$, and $-3 \lesssim c_{H_{d}} \lesssim 0$, which are compatible with the measured $\Delta a_{\mu}$ value within $2 \sigma$. Among them, $c_{5}$ and $c_{10}$ are important for having sizable $\Delta a_{\mu}$ by the light smuons. Even if the modulus-mediated contributions to the squarks are small, the squarks can be heavy due to the RG effects of heavy gluinos. The $c_{10}$ parameter is related to the mass and the mixing of top squarks. In our analysis result, it is mostly positive for achieving $m_{h} \simeq 125 \mathrm{GeV}$ without having too high SUSY-breaking scale. The $c_{H_{u}}$ parameter also plays an important role to have a stable vacuum as it affects the $\mu$ value through the condition of EW symmetry breaking. The negative values of $c_{H_{d}}$ is favored because it lifts up the slepton masses through mixed anomaly-modulus mediation and RG running effects. In appendix A, we list benchmark sparticle mass spectra.

Before closing this section, let us discuss the constraints from flavor-violating processes. We have calculated the flavor-violating observables by using SuperIso [85-87]. Figure 4 shows the scattering plot of $\mathrm{BR}\left(B_{s} \rightarrow \mu \mu\right)$ and $\mathrm{BR}(b \rightarrow s \gamma)$. The measured value of $\mathrm{BR}(b \rightarrow s \gamma)$ is $(3.32 \pm 0.15) \times 10^{-4}$ [88], and the SM prediction is $(3.36 \pm 0.23) \times 10^{-4}$ [89]. We refer to ref. [90] for the combined measurements of $\mathrm{BR}\left(B_{s} \rightarrow \mu \mu\right),(2.93 \pm 0.35) \times 10^{-9}$, and the SM prediction, $(3.67 \pm 0.15) \times 10^{-9}$. The colored regions in figure 4 are outside the $2 \sigma$ ranges from the experimental central values. The uncertainties have been obtained by quadrature sums of the SM and the experimental errors. The points with larger $\Delta a_{\mu}$ tend to have smaller SUSY contributions to the flavor-violating processes because the dangerous points with light sleptons and large $\tan \beta$ have already been excluded by requiring the vacuum stability condition. We have also checked that all the other flavor-violating observables calculated with SuperIso are consistent with the SM predictions within current uncertainties.

\section{Collider signatures and LHC constraints}

In this section, we discuss viable phenomenological scenarios of mixed modulus-anomaly mediation motivated by the muon $g-2$ anomaly and the relevant experimental constraints. Among the sparticles, in general, the colored sparticles receive the most severe constraints from the SUSY searches at hadron colliders. The latest LHC Run 2 analysis results of the ATLAS [91] and CMS [92] collaborations have excluded the gluino mass below $2.3 \mathrm{TeV}$. In our study, the $M_{0}$ value has been fixed by requiring the Higgs mass to be compatible with the measured SM-like Higgs mass, given the other model parameters. It results in a large value of $M_{0}$ that leads to heavy gluinos. Furthermore, as seen in section 2, a negative $\alpha$ can raise the gluino mass up to multi-TeV scales while leaving the EWinos around the weak scale. The upper left panel of figure 5 shows that $m_{\tilde{g}} \gtrsim 2.5 \mathrm{TeV}$ in the parameter space that can explain the muon $g-2$ anomaly within $2 \sigma$. On the other hand, the sfermion 
masses have a strong dependence on $M_{0}$ and $c_{i}$. In the parameter space for the muon $g-2$, the masses of the lighter stop are close to or slightly above the current lower limit, which is $m_{\tilde{t}_{1}} \gtrsim 1.2 \mathrm{TeV}[93,94]$. Consequently, we expect that the parameter points having stop masses around or above $1 \mathrm{TeV}$ will be tested by searches at the future LHC Run 3 and the High-Luminosity LHC. The other squark masses such as the lighter sbottom are well above the current experimental bounds. In appendix A, we present the benchmark sparticle mass spectra.

Contrary to the colored sparticles, the lighter chargino and neutralinos, as well as the sleptons, have masses around the weak scale to explain the muon $g-2$ anomaly. Therefore, the search results on the direct productions of the neutralinos/charginos and the sleptons can impose more serious limits on the parameter space than the experimental bounds discussed above. We exhibit the lighter chargino and slepton masses in figure 5. The lighter chargino is dominantly wino-like because the Higgsino is much heavier, $|\mu| \gtrsim 2 \mathrm{TeV}$, and hence the second lightest neutralino has degenerate mass with the chargino. The lefthanded sleptons tend to be heavier than the right-handed ones due to the RG effects from relatively large wino mass. The selectrons are nearly mass degenerate with the smuons, whereas the stau can have different masses than the other sleptons due to the left-right mixing terms and the RG effects.

Even though the muon $g-2$ anomaly hints at the existence of bino and smuons around the weak scale, it can lead to various phenomenological scenarios depending on the interactions and the mass spectrum of the sparticles in the low-energy scale. Classifying them by the property of the LOSP, we consider three phenomenological scenarios:

(1) The neutralino is the LOSP and is stable.

(2) The charged slepton is the LOSP and is metastable.

(3) The LOSP is unstable due to the RPV.

Here, being stable means that the particle does not necessarily be completely stable: it does not decay inside detectors at collider experiments.

\subsection{Stable neutralino LOSP}

In the first scenario, the LOSP is the lightest neutralino $\tilde{\chi}_{1}^{0}$. Because the Higgsino is very heavy, $|\mu| \gtrsim 2 \mathrm{TeV}$, in the parameter space for the muon $g-2, \tilde{\chi}_{1}^{0}$ is dominantly bino-like or an admixture of wino and bino. In this scenario, the most stringent limits come from the search results on the direct productions of the neutralino-chargino $\tilde{\chi}_{2}^{0} \tilde{\chi}_{1}^{ \pm}$ and the slepton pair $\tilde{\ell} \tilde{\ell}$ at the LHC [95-97]. Assuming mass-degenerate left-handed (righthanded) light flavor sleptons, the search results for the slepton pair productions have set the lower limit for the slepton mass to be $m_{\tilde{\ell}_{L}}>650 \mathrm{GeV}\left(m_{\tilde{\ell}_{R}}>500 \mathrm{GeV}\right)$ for $m_{\tilde{\chi}_{1}^{0}}$ being up to 400 (200) GeV [95]. Searches for stau pair productions can also give constraints because the stau is often the next-to-lightest SUSY particle in a portion of the parameter space with the neutralino LOSP that can explain the muon $g-2$ anomaly in our setup. However, the current limits for the staus decaying into the neutralino LOSP are not very 

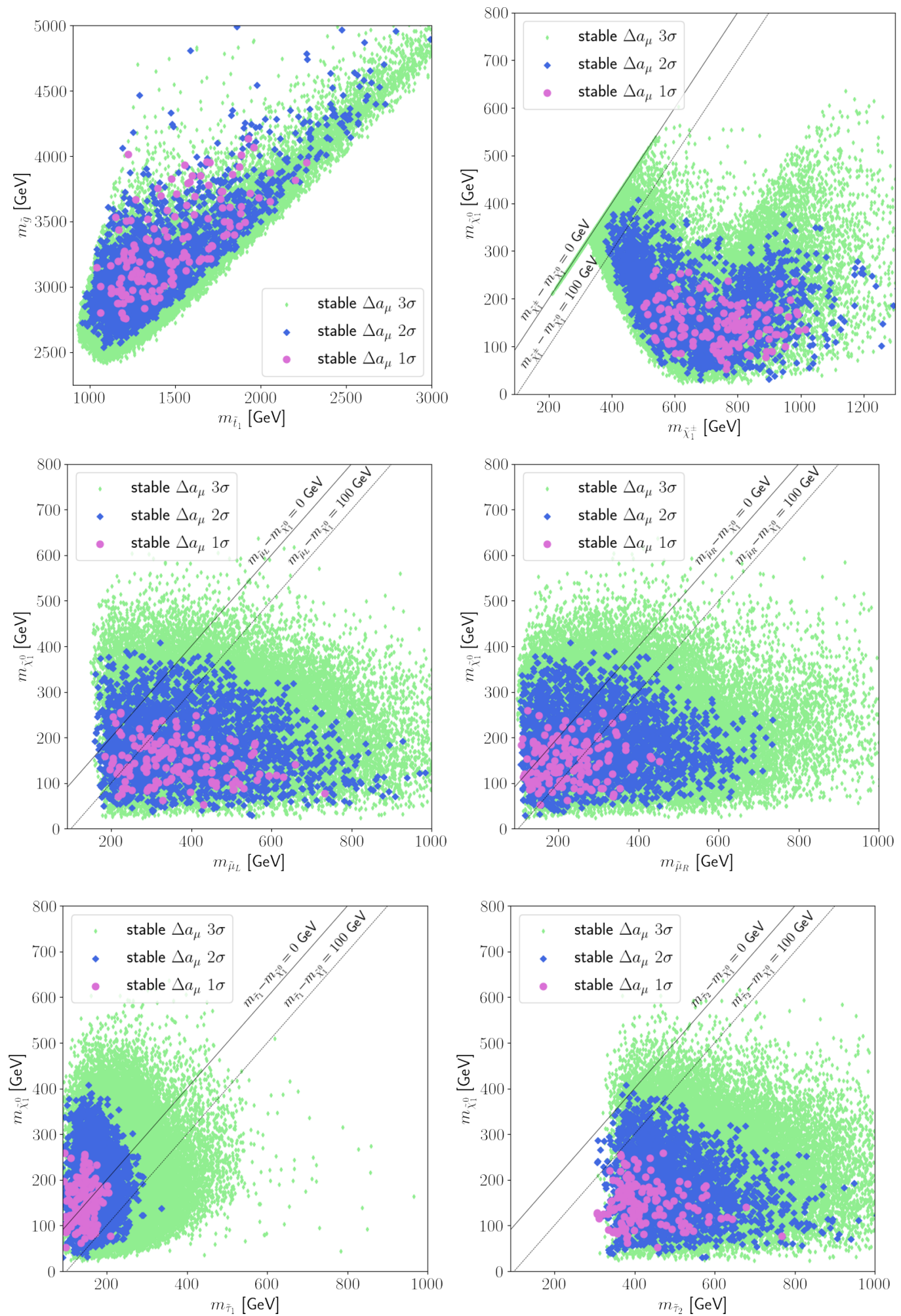

Figure 5. The masses of $\left(\tilde{t}_{1}, \tilde{g}\right)$ (upper left), ( $\left.\tilde{\chi}_{1}^{ \pm}, \tilde{\chi}_{1}^{0}\right)$ (upper right), $\left(\tilde{\mu}_{L}, \tilde{\chi}_{1}^{0}\right)$ (middle left), $\left(\tilde{\mu}_{R}\right.$, $\left.\tilde{\chi}_{1}^{0}\right)$ (middle right), $\left(\tilde{\tau}_{1}, \tilde{\chi}_{1}^{0}\right)$ (lower left), and $\left(\tilde{\tau}_{2}, \tilde{\chi}_{1}^{0}\right)$ (lower right) in $\mathrm{GeV}$ for the parameter points compatible with the muon $g-2$ anomaly. The color scheme is the same as in figure 3 . 
stringent, compared to those for the charginos and the other sleptons [98, 99]. A way out of the LHC constraints is to have sleptons mass-degenerate with the neutralino LOSP, $m_{\tilde{\ell}}-m_{\tilde{\chi}_{1}^{0}} \lesssim 80 \mathrm{GeV}$. The limits for such degenerate spectrum is quite restricted [100].

The search results on the neutralino-chargino production at the LHC set the stringent limits on the chargino mass $[95,96]$. If the mass gaps among the sleptons and the winos are sufficiently large, decays to all lepton flavors occur with almost equal probability, i.e., flavor-democratic decays. On the other hand, the wino-like states will dominantly decay into a stau and a tau (neutrino) if the other decay modes are kinematically forbidden. In the flavor-democratic case, the CMS analysis result for the integrated luminosity of $137 \mathrm{fb}^{-1}$ has excluded the wino-like chargino mass up to about $1.3 \mathrm{TeV}$ when $m_{\tilde{\chi}_{1}^{0}} \lesssim 800 \mathrm{GeV}[96]$. We see from figure 5 that, in the flavor-democratic case, all the parameter points are excluded by the CMS search result except for the mass-degenerate region. However, in the mass-degenerate region with $\alpha \lesssim-2.5$, the bino becomes heavy, and thus the sleptons must be light to explain $\Delta a_{\mu}$. Consequently, the flavor-democratic decays of the wino-like states are not achievable in the parameter region compatible with the muon $g-2$ anomaly. Meanwhile, if the wino-like states dominantly decay to a stau and a tau (neutrino), the lower limit for the chargino mass is about $800 \mathrm{GeV}$ for $m_{\tilde{\chi}_{1}^{0}} \lesssim 100 \mathrm{GeV}$ [96], which is much weaker than that in the flavor-democratic case. This can be realized if the winos are lighter than the left-handed selectron and smuon.

We conclude that, in the case of neutralino LOSP, the current LHC limits can be satisfied if the right-handed sleptons are nearly degenerate with the neutralino LOSP, and the left-handed selectron and smuon are sufficiently heavy so that the wino-like states dominantly decay to the stau: ${ }^{5}$

$$
m_{\tilde{\chi}_{1}^{0}} \lesssim m_{\tilde{\ell}_{R}}, \quad m_{\tilde{\chi}_{1}^{0}} \lesssim m_{\tilde{\tau}_{1}} \lesssim m_{\tilde{\chi}_{1}^{ \pm}, \tilde{\chi}_{2}^{0}} \lesssim m_{\tilde{e}_{L}, \tilde{\mu}_{L}}, \quad m_{\tilde{e}_{L}, \tilde{\mu}_{L}}>650 \mathrm{GeV} .
$$

In addition to the above mass hierarchy, one should ensure that the right-handed slepton is heavier than about $100 \mathrm{GeV}$ to avoid the lower limit from LEP on the slepton masses. The limit also applies indirectly to $m_{\tilde{\chi}_{1}^{0}}$ in the case where the bino-like neutralino LOSP is degenerate in mass with the slepton. Furthermore, if $m_{\tilde{\chi}_{1}^{0}} \lesssim 100 \mathrm{GeV}$, the lower limit from CMS on the chargino mass, $m_{\tilde{\chi}_{1}^{ \pm}} \gtrsim 800 \mathrm{GeV}$, should also be taken into account. The benchmark point A shown in appendix A corresponds to this scenario. It is interesting that the neutralino LOSP could serve as a good dark matter (DM) candidate through slepton co-annihilations due to the mass degeneracy. ${ }^{6}$

\subsection{Metastable slepton LOSP}

If the slepton is the LOSP, there should be lighter sparticle than it or R-parity should be violated so that the slepton LOSP can decay. The latter scenario will be discussed in

\footnotetext{
${ }^{5}$ Here, we have used the "§" symbol to indicate that the two particles are close in mass while evading the LHC limits by following the notation in ref. [43].

${ }^{6}$ The bino-like neutralino LOSP around the weak scale may be overproduced via late-time decays of a modulus [101] unless the modulus is located quite close to the potential minimum after the primordial inflation. This fact also motivates us to consider the axino as the lightest SUSY particle (LSP) or RPV scenarios, which will be discussed in the following subsections.
} 
the next subsection. In the former case, a scenario worth considering is a PQ symmetric extension where the axion solves the strong $\mathrm{CP}$ problem, and the axino $\tilde{a}$ contributes to the DM [102]. For instance, if the saxion is radiatively stabilized [103, 104], the axino naturally becomes the LSP because its mass is one-loop suppressed compared to other sparticle masses. The scenario is noteworthy because, in a majority of the parameter space compatible with the muon $g-2$ anomaly, we find that a slepton is lighter than the lightest neutralino: the stau is the LOSP in more than half of the parameter space and the selectron or the smuon is the LOSP in many other parameter points. Then, the slepton LOSP will mainly undergo the two-body decay, $\tilde{\ell} \rightarrow \ell \tilde{a}$.

In this scenario, the slepton LOSP becomes a heavy stable charged particle (HSCP), mostly decaying outside the detector, because it can have a lifetime longer than $10^{4} \mathrm{~ns}$ to a few hundred seconds, depending on the axion decay constant and the masses of the involved sparticle masses [105]. Due to the long lifetime, the scenario is not constrained by the search results for displaced leptons because it is sensitive to the particles with lifetime shorter than 1 ns [106]. At LEP2, the null detection of the HSCPs set the lower mass limit of about $100 \mathrm{GeV}$ [107]. In recent years, the constraint has been updated further by the searches for HSCPs at the LHC. In particular, the CMS collaboration performed model-independent analyses for various possible HSCPs and excluded stau masses below $360 \mathrm{GeV}$ [108]. In the scenario with the axino LSP, the CMS limit can impose a serious impact on our analysis result because the lighter stau is lighter than about $350 \mathrm{GeV}$ in the parameter points with the stau LOSP for the muon $g-2$ anomaly within $3 \sigma$, as can be seen in the lower left panel of figure 5.

\subsection{Unstable LOSP}

The LOSP, either neutralino or slepton, decays to SM particles if the RPV interactions are allowed. In the RPV scenario, the axion can serve as a candidate for the DM [109]. The relevant RPV terms in the superpotential are given as follows:

$$
W \supset \frac{1}{2} \lambda_{i j k} L_{i} L_{j} \bar{E}_{k}+\lambda_{i j k}^{\prime} L_{i} Q_{j} \bar{D}_{k}+\frac{1}{2} \lambda_{i j k}^{\prime \prime} \bar{U}_{i} \bar{D}_{j} \bar{D}_{k}
$$

where $i, j, k$ are flavor indices. The $\lambda_{i j k}$ and $\lambda_{i j k}^{\prime}$ terms violate lepton number while the $\lambda_{i j k}^{\prime \prime}$ terms violate baryon number. Either of the lepton or baryon number conservation should hold with good accuracy to avoid too fast proton decay.

If the terms with the $\lambda_{i j k}$ couplings are dominant among the others, the slepton LOSP will mainly decay into a charged lepton and a neutrino, $\tilde{\ell} \rightarrow \ell_{j} \nu_{\ell_{k}}$. The slepton pair production then gives rise to the signature of $2 \ell+E_{T}^{\text {miss }}$. The signature is similar to that of the R-parity conserving case, and it receives the bounds from the aforementioned SUSY searches for multi-lepton final states. Recasting of the LHC search results has revealed that the lower limit of the stau LOSP is about $225 \mathrm{GeV}$ in the case where the stau is right-handed [110]. A large portion of our parameter space could be excluded by the limit. Meanwhile, if either $\lambda_{i j k}^{\prime}$ or $\lambda_{i j k}^{\prime \prime}$ term is dominant, the final states of the slepton decays are quite different. In the case where the $\lambda_{i j k}^{\prime}$ operators are dominant, the slepton LOSP can decay into the final state of two leptons + two quark-jets via four-body processes. For 
example, the stau LOSP can decay as

$$
\tilde{\tau}_{1} \rightarrow \tau+\tilde{\chi}_{1}^{0 *} \rightarrow \tau+\mu u \bar{d}
$$

via the $\lambda_{211}^{\prime}$ operator. See refs. $[111,112]$ for a list of possible LHC signatures. The decay length of the stau LOSP can be $\mathcal{O}\left(10^{-6}\right) \mathrm{m}$ for $\lambda^{\prime} \simeq 10^{-3}$ and $m_{\tilde{\tau}_{1}} \simeq m_{\tilde{\chi}_{1}^{0}} \simeq 100 \mathrm{GeV}$, resulting in displaced vertices [113]. As the gluino and squark masses are beyond the current experimental limits, the slepton LOSP could be produced via the neutralino-chargino or the direct slepton pair processes. The signatures have not yet been covered by the LHC searches so far. Therefore, we conclude that the slepton LOSP scenario with RPV would be viable unless the $\lambda_{i j k}$ operator is the dominant RPV interaction.

In the case of neutralino LOSP with RPV, we can reach a similar conclusion. If Rparity is violated dominantly by the $\lambda_{i j k}$ coupling, there are strong constraints due to the signatures of high-multiplicity leptons $[114,115]$. For instance, the limits for sleptons and charginos are about 800 and $1000 \mathrm{GeV}$, respectively, in the scenario of nonzero $\lambda_{i 33}$ [114]. In the other cases where either $\lambda_{i j k}^{\prime}$ or $\lambda_{i j k}^{\prime \prime}$ is dominant, the limits are much weaker or absent.

\section{Summary}

Since the new measurement of the muon $g-2$ at the Fermilab experiment, physicists have regained attention on the existence of new physics in the lepton sector. If new physics responsible for the muon $g-2$ anomaly is supersymmetric, one should consider how to obtain light EWinos and sleptons in a systematic way. Combined with the gauge coupling unification, the gaugino masses exhibit a robust pattern controlled by a single parameter $\alpha$ that represents the size of anomaly mediation. The EWinos can be much lighter than the gluino if $\alpha$ is negative and of order unity, as is required to explain the muon $g-2$ anomaly while avoiding experimental constraints. The KKLT provides a natural and interesting framework for such mixed mediation, where the pattern of gaugino masses is determined by $\alpha$, while that of sfermion masses depends on how the corresponding matter field couples to the string moduli sector.

We have performed a numerical analysis to explore the parameter space of mixed modulus-anomaly mediation realized in the generalized KKLT setup and identified the parameter region compatible with the muon $g-2$ anomaly. To have light EWinos, it is essential to construct a setup of KKLT moduli stabilization yielding a negative $\alpha$. As a byproduct, it can make the gluino heavier than a few $\mathrm{TeV}$, thus we can easily evade the lower limit of gluino at the LHC. On the other hand, due to light sleptons, imposing the condition of vacuum stability of the scalar potential is crucial, and it excludes the parameter space of large $\tan \beta \gtrsim 30$.

In the viable parameter region, we find that the LOSP can be either bino-like neutralino or slepton. However, in the case of the neutralino LOSP, the slepton and charginoneutralino searches at the LHC exclude a vast parameter space of the R-parity conservation. The current LHC limits can be satisfied only when the mass spectrum of eq. (4.1) is realized. In most cases, the wino cannot be sufficiently heavy or degenerate with the bino as far as the sleptons are sufficiently light due to the gaugino mass relations predicted in 
the mixed modulus-anomaly mediation. To avoid this difficulty, one may consider a more general case with $c_{i} \neq a_{i}$. Another way is to add gauge-mediated contributions so that the deflection of sparticle masses occurs at the gauge-messenger scale [116, 117]. Meanwhile, when a slepton is lighter than the neutralinos, we should consider alternative scenarios such as axino LSP or RPV interactions. In the former case with axino LSP, the lightest slepton becomes long-lived and will decay outside the detector. The recent CMS result on long-lived charged particles has excluded such possibility. On the other side, the RPV interactions with either lepton or baryon number violation can be a viable option because of unexplored signatures with the final states of multi-jets and -leptons with small or no missing energy at the LHC.

\section{Acknowledgments}

This work was supported by the National Research Foundation of Korea (NRF) grant funded by the Korean government NRF-2018R1C1B6006061 (K.S.J.), the Institute for Basic Science (IBS) under the project code IBS-R018-D1 (J.K. and C.B.P.), and the Grantin-Aid for Scientific Research from the Ministry of Education, Science, Sports and Culture (MEXT), Japan No. 18K13534 (J.K.).

\section{A Benchmark sparticle mass spectrum}

The sparticle mass spectra of our benchmark points in mixed modulus-anomaly mediation are shown in table 1. At points A and B, the LOSP is bino-like, while it is the lightest stau at points $\mathrm{C}$ and $\mathrm{D}$. The sign of bino mass $M_{1}$ is taken to be positive at points $\mathrm{A}$ and $\mathrm{C}$, while it is negative at points $\mathrm{B}$ and $\mathrm{D}$. At points $\mathrm{A}$ and $\mathrm{B}$, the right-handed sleptons are degenerate with the lightest neutralino, and the left-handed ones are sufficiently heavy so that the current limits can be evaded. Point A, which realizes the mass spectra of eq. (4.1), can be safe from the constraint from the latest CMS search results on the chargino-neutralino productions because the stau is lighter than the wino-like states, while the other left-handed sleptons are heavier. In this case, $\tilde{\chi}_{1}^{ \pm}$and $\tilde{\chi}_{2}^{0}$ dominantly decay into the stau and the tau (neutrino). Meanwhile, point B is excluded by the CMS search result because the wino-like states decay into the sleptons with nearly equal branching fraction to each flavor. Points $\mathrm{C}$ and D could be excluded by the HSCP searches at the LHC if the stau is metastable. However, if R-parity is violated mainly by $\lambda^{\prime}$ or $\lambda^{\prime \prime}$, all the points are still viable and can be searched at the LHC or future colliders through the final states of multi-leptons and jets. 


\begin{tabular}{|c|c|c|c|c|}
\hline & $\mathrm{A}$ & B & $\mathrm{C}$ & $\mathrm{D}$ \\
\hline $\tan \beta$ & 30.41 & 9.955 & 23.63 & 12.9 \\
\hline $\operatorname{sgn}(\mu)$ & 1 & -1 & 1 & -1 \\
\hline$M_{0}$ & 1038 & 1062 & 1250 & 1344 \\
\hline$\alpha$ & -0.7734 & -1.51 & -0.5258 & -1.745 \\
\hline$c_{Q}$ & 0.06998 & 0.1594 & 0.1025 & 0.05333 \\
\hline$c_{U}$ & 0.06998 & 0.1594 & 0.1025 & 0.05333 \\
\hline$c_{D}$ & -0.1951 & -0.8861 & -0.5709 & -0.8574 \\
\hline$c_{L}$ & -0.1951 & -0.8861 & -0.5709 & -0.8574 \\
\hline$c_{E}$ & 0.06998 & 0.1594 & 0.1025 & 0.05333 \\
\hline$c_{H_{u}}$ & 2.892 & 3.829 & 2.312 & 2.373 \\
\hline$c_{H_{d}}$ & -1.665 & -0.9771 & -0.9358 & -0.899 \\
\hline$m_{h}$ & 125.1 & 125.1 & 125.1 & 125.1 \\
\hline$m_{A}$ & 1735 & 3021 & 1952 & 3451 \\
\hline$m_{\tilde{g}}$ & 2864 & 3507 & 3175 & 4584 \\
\hline$m_{\tilde{\chi}_{1}^{0}}$ & 157.9 & 120.1 & 301.7 & 262 \\
\hline$m_{\tilde{\chi}_{2}^{0}}$ & 767.8 & 704.4 & 950.1 & 861.2 \\
\hline$m_{\tilde{\chi}_{1}^{ \pm}}$ & 768 & 704.5 & 950.2 & 861.4 \\
\hline$m_{\tilde{\chi}_{3}^{0}}$ & 2062 & 3021 & 2111 & 3453 \\
\hline$m_{\tilde{\chi}_{4}^{0}}$ & 2064 & 3021 & 2113 & 3453 \\
\hline$m_{\tilde{\chi}_{2}^{ \pm}}$ & 2065 & 3022 & 2114 & 3454 \\
\hline$m_{\tilde{b}_{1}}$ & 2133 & 2579 & 2427 & 3649 \\
\hline$m_{\tilde{b}_{2}}$ & 2487 & 3040 & 2624 & 3990 \\
\hline$m_{\tilde{t}_{1}}$ & 1304 & 1237 & 1618 & 2600 \\
\hline$m_{\tilde{t}_{2}}$ & 2160 & 2597 & 2451 & 3661 \\
\hline$m_{\tilde{q}_{1}}$ & 2698 & 3364 & 2984 & 4376 \\
\hline$m_{\tilde{u}_{1}}$ & 2639 & 3290 & 2907 & 4262 \\
\hline$m_{\tilde{d}_{1}}$ & 2526 & 3049 & 2655 & 4014 \\
\hline$m_{\tilde{\tau}_{1}}$ & 182.3 & 144.3 & 265.2 & 244.5 \\
\hline$m_{\tilde{\tau}_{2}}$ & 796.1 & 374.2 & 497 & 449.3 \\
\hline$m_{\tilde{\mu}_{L}}$ & 775.4 & 213.7 & 367.8 & 346.5 \\
\hline$m_{\tilde{\mu}_{R}}$ & 163.7 & 334.2 & 416.3 & 350.5 \\
\hline$m_{\tilde{e}_{L}}$ & 775.3 & 213.6 & 367.8 & 346.4 \\
\hline$m_{\tilde{e}_{R}}$ & 163.4 & 334.2 & 416.2 & 350.4 \\
\hline$m_{\tilde{\nu}_{e}}$ & 778.3 & 203.3 & 364.3 & 346.9 \\
\hline$m_{\tilde{\nu}_{\mu}}$ & 771.1 & 199.1 & 359.2 & 337.4 \\
\hline$m_{\tilde{\nu}_{\tau}}$ & 771.1 & 199.1 & 359.2 & 337.3 \\
\hline$\Delta a_{\mu} \times 10^{9}$ & 1.643 & 2.275 & 1.442 & 1.433 \\
\hline $\mathrm{BR}(b \rightarrow s \gamma) \times 10^{4}$ & 3.177 & 3.517 & 3.284 & 3.461 \\
\hline $\mathrm{BR}\left(B_{s} \rightarrow \mu \mu\right) \times 10^{9}$ & 3.862 & 3.216 & 3.418 & 3.213 \\
\hline$\eta_{\mu}$ & 0.0653 & 0.0562 & 0.0546 & 0.0592 \\
\hline$\eta_{\tau}$ & 0.8674 & 0.8910 & 0.8683 & 0.9077 \\
\hline LOSP & $\tilde{\chi}_{1}^{0}$ & $\tilde{\chi}_{1}^{0}$ & $\overline{\tilde{\tau}_{1}}$ & $\overline{\tilde{\tau}_{1}}$ \\
\hline
\end{tabular}

Table 1. Benchmark sparticle mass spectra of mixed modulus-anomaly mediation for the muon $g-2$ anomaly. 
Open Access. This article is distributed under the terms of the Creative Commons Attribution License (CC-BY 4.0), which permits any use, distribution and reproduction in any medium, provided the original author(s) and source are credited.

\section{References}

[1] Muon G-2 collaboration, Measurement of the negative muon anomalous magnetic moment to 0.7 ppm, Phys. Rev. Lett. 92 (2004) 161802 [hep-ex/0401008] [INSPIRE].

[2] Muon G-2 collaboration, Measurement of the positive muon anomalous magnetic moment to 0.46 ppm, Phys. Rev. Lett. 126 (2021) 141801 [arXiv:2104.03281] [INSPIRE].

[3] A. Czarnecki, W.J. Marciano and A. Vainshtein, Refinements in electroweak contributions to the muon anomalous magnetic moment, Phys. Rev. D 67 (2003) 073006 [Erratum ibid. 73 (2006) 119901] [hep-ph/0212229] [INSPIRE].

[4] K. Melnikov and A. Vainshtein, Hadronic light-by-light scattering contribution to the muon anomalous magnetic moment revisited, Phys. Rev. D 70 (2004) 113006 [hep-ph/0312226] [INSPIRE].

[5] T. Aoyama, M. Hayakawa, T. Kinoshita and M. Nio, Complete tenth-order QED contribution to the muon g-2, Phys. Rev. Lett. 109 (2012) 111808 [arXiv:1205.5370] [INSPIRE].

[6] C. Gnendiger, D. Stöckinger and H. Stöckinger-Kim, The electroweak contributions to $(g-2)_{\mu}$ after the Higgs boson mass measurement, Phys. Rev. D 88 (2013) 053005 [arXiv: 1306.5546] [INSPIRE].

[7] A. Kurz, T. Liu, P. Marquard and M. Steinhauser, Hadronic contribution to the muon anomalous magnetic moment to next-to-next-to-leading order, Phys. Lett. B 734 (2014) 144 [arXiv:1403.6400] [INSPIRE].

[8] G. Colangelo, M. Hoferichter, A. Nyffeler, M. Passera and P. Stoffer, Remarks on higher-order hadronic corrections to the muon g-2, Phys. Lett. B 735 (2014) 90 [arXiv: 1403.7512] [INSPIRE].

[9] P. Masjuan and P. Sanchez-Puertas, Pseudoscalar-pole contribution to the $\left(g_{\mu}-2\right)$ : a rational approach, Phys. Rev. D 95 (2017) 054026 [arXiv:1701.05829] [INSPIRE].

[10] G. Colangelo, M. Hoferichter, M. Procura and P. Stoffer, Dispersion relation for hadronic light-by-light scattering: two-pion contributions, JHEP 04 (2017) 161 [arXiv:1702.07347] [INSPIRE].

[11] M. Davier, A. Hoecker, B. Malaescu and Z. Zhang, Reevaluation of the hadronic vacuum polarisation contributions to the Standard Model predictions of the muon $g-2$ and $\alpha\left(m_{Z}^{2}\right)$ using newest hadronic cross-section data, Eur. Phys. J. C 77 (2017) 827 [arXiv: 1706. 09436] [INSPIRE].

[12] A. Keshavarzi, D. Nomura and T. Teubner, Muon $g-2$ and $\alpha\left(M_{Z}^{2}\right)$ : a new data-based analysis, Phys. Rev. D 97 (2018) 114025 [arXiv:1802.02995] [inSPIRE].

[13] M. Hoferichter, B.-L. Hoid, B. Kubis, S. Leupold and S.P. Schneider, Dispersion relation for hadronic light-by-light scattering: pion pole, JHEP 10 (2018) 141 [arXiv:1808.04823] [INSPIRE].

[14] G. Colangelo, M. Hoferichter and P. Stoffer, Two-pion contribution to hadronic vacuum polarization, JHEP 02 (2019) 006 [arXiv:1810.00007] [INSPIRE]. 
[15] T. Aoyama, T. Kinoshita and M. Nio, Theory of the anomalous magnetic moment of the electron, Atoms 7 (2019) 28 [inSPIRE].

[16] A. Gérardin, H.B. Meyer and A. Nyffeler, Lattice calculation of the pion transition form factor with $N_{f}=2+1$ Wilson quarks, Phys. Rev. D 100 (2019) 034520 [arXiv: 1903.09471] [INSPIRE].

[17] M. Hoferichter, B.-L. Hoid and B. Kubis, Three-pion contribution to hadronic vacuum polarization, JHEP 08 (2019) 137 [arXiv:1907.01556] [INSPIRE].

[18] M. Davier, A. Hoecker, B. Malaescu and Z. Zhang, A new evaluation of the hadronic vacuum polarisation contributions to the muon anomalous magnetic moment and to $\alpha\left(m_{Z}^{2}\right)$, Eur. Phys. J. C 80 (2020) 241 [Erratum ibid. 80 (2020) 410] [arXiv:1908.00921] [INSPIRE].

[19] J. Bijnens, N. Hermansson-Truedsson and A. Rodríguez-Sánchez, Short-distance constraints for the HLbL contribution to the muon anomalous magnetic moment, Phys. Lett. B 798 (2019) 134994 [arXiv:1908.03331] [INSPIRE].

[20] G. Colangelo, F. Hagelstein, M. Hoferichter, L. Laub and P. Stoffer, Longitudinal short-distance constraints for the hadronic light-by-light contribution to $(g-2)_{\mu}$ with large- $N_{c}$ Regge models, JHEP 03 (2020) 101 [arXiv: 1910.13432] [INSPIRE].

[21] A. Keshavarzi, D. Nomura and T. Teubner, $g-2$ of charged leptons, $\alpha\left(M_{Z}^{2}\right)$, and the hyperfine splitting of muonium, Phys. Rev. D 101 (2020) 014029 [arXiv:1911.00367] [INSPIRE].

[22] T. Blum et al., Hadronic light-by-light scattering contribution to the muon anomalous magnetic moment from lattice QCD, Phys. Rev. Lett. 124 (2020) 132002 [arXiv: 1911.08123] [INSPIRE].

[23] T. Aoyama et al., The anomalous magnetic moment of the muon in the Standard Model, Phys. Rept. 887 (2020) 1 [arXiv: 2006. 04822] [INSPIRE].

[24] S. Borsányi et al., Leading hadronic contribution to the muon magnetic moment from lattice QCD, Nature 593 (2021) 51 [arXiv:2002.12347] [INSPIRE].

[25] C. Lehner and A.S. Meyer, Consistency of hadronic vacuum polarization between lattice QCD and the R-ratio, Phys. Rev. D 101 (2020) 074515 [arXiv:2003.04177] [INSPIRE].

[26] A. Crivellin, M. Hoferichter, C.A. Manzari and M. Montull, Hadronic vacuum polarization: $(g-2)_{\mu}$ versus global electroweak fits, Phys. Rev. Lett. 125 (2020) 091801 [arXiv:2003.04886] [INSPIRE].

[27] A. Keshavarzi, W.J. Marciano, M. Passera and A. Sirlin, Muon $g-2$ and $\Delta \alpha$ connection, Phys. Rev. D 102 (2020) 033002 [arXiv: 2006.12666] [inSPIRE].

[28] B. Malaescu and M. Schott, Impact of correlations between $a_{\mu}$ and $\alpha_{Q E D}$ on the $E W$ fit, Eur. Phys. J. C 81 (2021) 46 [arXiv:2008.08107] [InSPIRE].

[29] A. Crivellin and M. Hoferichter, Consequences of chirally enhanced explanations of $(g-2)_{\mu}$ for $h \rightarrow \mu \mu$ and $Z \rightarrow \mu \mu$, JHEP 07 (2021) 135 [arXiv: 2104.03202] [INSPIRE].

[30] M. Endo, K. Hamaguchi, S. Iwamoto and T. Kitahara, Supersymmetric interpretation of the muon $g-2$ anomaly, JHEP 07 (2021) 075 [arXiv: 2104.03217] [INSPIRE].

[31] Y. Gu, N. Liu, L. Su and D. Wang, Heavy bino and slepton for muon $g-2$ anomaly, Nucl. Phys. B 969 (2021) 115481 [arXiv: 2104.03239] [INSPIRE].

[32] M. Van Beekveld, W. Beenakker, M. Schutten and J. De Wit, Dark matter, fine-tuning and $(g-2)_{\mu}$ in the pMSSM, SciPost Phys. 11 (2021) 049 [arXiv:2104.03245] [INSPIRE]. 
[33] W. Yin, Muon g-2 anomaly in anomaly mediation, JHEP 06 (2021) 029 [arXiv: 2104.03259] [INSPIRE].

[34] F. Wang, L. Wu, Y. Xiao, J.M. Yang and Y. Zhang, GUT-scale constrained SUSY in light of new muon g-2 measurement, Nucl. Phys. B 970 (2021) 115486 [arXiv:2104.03262] [INSPIRE].

[35] M. Abdughani, Y.-Z. Fan, L. Feng, Y.-L.S. Tsai, L. Wu and Q. Yuan, A common origin of muon $g-2$ anomaly, Galaxy Center GeV excess and AMS-02 anti-proton excess in the NMSSM, Sci. Bull. 66 (2021) 1545 [arXiv:2104.03274] [inSPIRE].

[36] M. Ibe, S. Kobayashi, Y. Nakayama and S. Shirai, Muon $g-2$ in gauge mediation without SUSY CP problem, arXiv:2104.03289 [INSPIRE].

[37] P. Cox, C. Han and T.T. Yanagida, Muon $g-2$ and co-annihilating dark matter in the $M S S M$, arXiv:2104.03290 [INSPIRE].

[38] C. Han, Muon $g-2$ and CP-violation in MSSM, arXiv:2104.03292 [INSPIRE].

[39] S. Baum, M. Carena, N.R. Shah and C.E.M. Wagner, The tiny $(g-2)$ muon wobble from small- $\mu$ supersymmetry, arXiv:2104.03302 [INSPIRE].

[40] H.-B. Zhang, C.-X. Liu, J.-L. Yang and T.-F. Feng, Muon anomalous magnetic dipole moment in the $\mu \nu S S M$, arXiv:2104.03489 [INSPIRE].

[41] W. Ahmed, I. Khan, J. Li, T. Li, S. Raza and W. Zhang, The natural explanation of the muon anomalous magnetic moment via the electroweak supersymmetry from the GmSUGRA in the MSSM, arXiv:2104.03491 [INSPIRE].

[42] J.-L. Yang, H.-B. Zhang, C.-X. Liu, X.-X. Dong and T.-F. Feng, Muon $(g-2)$ in the $B$-LSSM, arXiv:2104.03542 [INSPIRE].

[43] P. Athron, C. Balázs, D.H. Jacob, W. Kotlarski, D. Stöckinger and H. Stöckinger-Kim, New physics explanations of $a_{\mu}$ in light of the FNAL muon $g-2$ measurement, arXiv:2104.03691 [INSPIRE].

[44] A. Aboubrahim, M. Klasen and P. Nath, What the Fermilab muon g-2 experiment tells us about discovering supersymmetry at high luminosity and high energy upgrades to the LHC, Phys. Rev. D 104 (2021) 035039 [arXiv:2104.03839] [INSPIRE].

[45] M. Chakraborti, L. Roszkowski and S. Trojanowski, GUT-constrained supersymmetry and dark matter in light of the new $(g-2)_{\mu}$ determination, JHEP 05 (2021) 252 [arXiv:2104.04458] [INSPIRE].

[46] H. Baer, V. Barger and H. Serce, Anomalous muon magnetic moment, supersymmetry, naturalness, LHC search limits and the landscape, Phys. Lett. B 820 (2021) 136480 [arXiv:2104.07597] [INSPIRE].

[47] W. Altmannshofer, S.A. Gadam, S. Gori and N. Hamer, Explaining $(g-2)_{\mu}$ with multi-TeV sleptons, arXiv:2104.08293 [INSPIRE].

[48] A. Aboubrahim, P. Nath and R.M. Syed, Yukawa coupling unification in an $\mathrm{SO}(10)$ model consistent with Fermilab $(g-2)_{\mu}$ result, JHEP 06 (2021) 002 [arXiv:2104.10114] [INSPIRE].

[49] M. Chakraborti, S. Heinemeyer and I. Saha, Improved $(g-2)_{\mu}$ Measurements and Supersymmetry : Implications for $e^{+} e^{-}$colliders, arXiv:2105.06408 [INSPIRE].

[50] M.-D. Zheng and H.-H. Zhang, Studying the $b \rightarrow s \ell^{+} \ell^{-}$anomalies and $(g-2)_{\mu}$ in RPV-MSSM framework with inverse seesaw, arXiv:2105.06954 [INSPIRE]. 
[51] Z.-N. Zhang, H.-B. Zhang, J.-L. Yang, S.-M. Zhao and T.-F. Feng, Higgs boson decays with lepton flavor violation in the B-L symmetric SSM, Phys. Rev. D 103 (2021) 115015 [arXiv: 2105. 09799] [INSPIRE].

[52] D. Stöckinger, The muon magnetic moment and supersymmetry, J. Phys. G 34 (2007) R45 [hep-ph/0609168] [INSPIRE].

[53] K. Choi and H.P. Nilles, The gaugino code, JHEP 04 (2007) 006 [hep-ph/0702146] [INSPIRE].

[54] H.P. Nilles, Dynamically broken supergravity and the hierarchy problem, Phys. Lett. B 115 (1982) 193 [INSPIRE].

[55] M. Dine, W. Fischler and M. Srednicki, Supersymmetric technicolor, Nucl. Phys. B 189 (1981) 575 [INSPIRE].

[56] S. Dimopoulos and S. Raby, Supercolor, Nucl. Phys. B 192 (1981) 353 [InSPIRE].

[57] M. Dine and W. Fischler, A phenomenological model of particle physics based on supersymmetry, Phys. Lett. B 110 (1982) 227 [INSPIRE].

[58] L. Randall and R. Sundrum, Out of this world supersymmetry breaking, Nucl. Phys. B 557 (1999) 79 [hep-th/9810155] [INSPIRE].

[59] G.F. Giudice, M.A. Luty, H. Murayama and R. Rattazzi, Gaugino mass without singlets, JHEP 12 (1998) 027 [hep-ph/9810442] [InSPIRE].

[60] S. Kachru, R. Kallosh, A.D. Linde and S.P. Trivedi, De Sitter vacua in string theory, Phys. Rev. D 68 (2003) 046005 [hep-th/0301240] [INSPIRE].

[61] K. Choi, A. Falkowski, H.P. Nilles, M. Olechowski and S. Pokorski, Stability of flux compactifications and the pattern of supersymmetry breaking, JHEP 11 (2004) 076 [hep-th/0411066] [INSPIRE].

[62] K. Choi, A. Falkowski, H.P. Nilles and M. Olechowski, Soft supersymmetry breaking in KKLT flux compactification, Nucl. Phys. B 718 (2005) 113 [hep-th/0503216] [INSPIRE].

[63] M. Endo, M. Yamaguchi and K. Yoshioka, A Bottom-up approach to moduli dynamics in heavy gravitino scenario: superpotential, soft terms and sparticle mass spectrum, Phys. Rev. $D 72$ (2005) 015004 [hep-ph/0504036] [INSPIRE].

[64] K. Choi, K.S. Jeong and K.-i. Okumura, Phenomenology of mixed modulus-anomaly mediation in fluxed string compactifications and brane models, JHEP 09 (2005) 039 [hep-ph/0504037] [INSPIRE].

[65] A. Falkowski, O. Lebedev and Y. Mambrini, SUSY phenomenology of KKLT flux compactifications, JHEP 11 (2005) 034 [hep-ph/0507110] [INSPIRE].

[66] R. Kitano and Y. Nomura, A solution to the supersymmetric fine-tuning problem within the MSSM, Phys. Lett. B 631 (2005) 58 [hep-ph/0509039] [INSPIRE].

[67] O. Lebedev, H.P. Nilles and M. Ratz, A note on fine-tuning in mirage mediation, hep-ph/0511320 [INSPIRE].

[68] K. Choi, K.S. Jeong, T. Kobayashi and K.-i. Okumura, TeV scale mirage mediation and natural little SUSY hierarchy, Phys. Rev. D 75 (2007) 095012 [hep-ph/0612258] [InSPIRE].

[69] H. Abe and J. Kawamura, The $126 \mathrm{GeV}$ Higgs boson mass and naturalness in (deflected) mirage mediation, JHEP 07 (2014) 077 [arXiv: 1405.0779] [INSPIRE].

[70] H. Baer, V. Barger, H. Serce and X. Tata, Natural generalized mirage mediation, Phys. Rev. D 94 (2016) 115017 [arXiv: 1610.06205] [inSPIRE]. 
[71] J. Kawamura and Y. Omura, Analysis of the TeV-scale mirage mediation with heavy superparticles, JHEP 11 (2017) 189 [arXiv:1710.03412] [INSPIRE].

[72] K.S. Jeong and C.B. Park, Light higgsinos for electroweak naturalness in mirage-mediated high-scale supersymmetry, arXiv:2011.11993 [INSPIRE].

[73] X.K. Du, G.-L. Liu, F. Wang, W. Wang, J.M. Yang and Y. Zhang, NMSSM with generalized deflected mirage mediation, Eur. Phys. J. C 79 (2019) 397 [arXiv: 1804.07335] [InSPIRE].

[74] ATLAS, CMS collaboration, Combined measurement of the Higgs boson mass in pp collisions at $\sqrt{s}=7$ and $8 \mathrm{TeV}$ with the ATLAS and CMS experiments, Phys. Rev. Lett. 114 (2015) 191803 [arXiv: 1503.07589] [INSPIRE].

[75] H. Abe, T. Higaki and T. Kobayashi, KKLT type models with moduli-mixing superpotential, Phys. Rev. D 73 (2006) 046005 [hep-th/0511160] [INSPIRE].

[76] M.B. Green and J.H. Schwarz, Anomaly cancellation in supersymmetric D $=10$ gauge theory and superstring theory, Phys. Lett. B 149 (1984) 117 [InSPIRE].

[77] K. Choi and K.S. Jeong, Supersymmetry breaking and moduli stabilization with anomalous U(1) gauge symmetry, JHEP 08 (2006) 007 [hep-th/0605108] [INSPIRE].

[78] C.D. Froggatt and H.B. Nielsen, Hierarchy of quark masses, Cabibbo angles and CP-violation, Nucl. Phys. B 147 (1979) 277 [InSPIRE].

[79] P.Z. Skands et al., SUSY Les Houches accord: interfacing SUSY spectrum calculators, decay packages, and event generators, JHEP 07 (2004) 036 [hep-ph/0311123] [INSPIRE].

[80] B.C. Allanach, SOFTSUSY: a program for calculating supersymmetric spectra, Comput. Phys. Commun. 143 (2002) 305 [hep-ph/0104145] [InSPIRE].

[81] P. Athron et al., GM2Calc: precise MSSM prediction for $(g-2)$ of the muon, Eur. Phys. J. C 76 (2016) 62 [arXiv:1510.08071] [inSPIRE].

[82] M. Endo, K. Hamaguchi, T. Kitahara and T. Yoshinaga, Probing bino contribution to muon $g-2$, JHEP 11 (2013) 013 [arXiv:1309.3065] [INSPIRE].

[83] T. Kitahara and T. Yoshinaga, Stau with large mass difference and enhancement of the Higgs to diphoton decay rate in the MSSM, JHEP 05 (2013) 035 [arXiv:1303.0461] [INSPIRE].

[84] G.-C. Cho, K. Hagiwara, Y. Matsumoto and D. Nomura, The MSSM confronts the precision electroweak data and the muon g-2, JHEP 11 (2011) 068 [arXiv:1104.1769] [INSPIRE].

[85] F. Mahmoudi, SuperIso: a program for calculating the isospin asymmetry of $B \rightarrow K^{*} \gamma$ in the MSSM, Comput. Phys. Commun. 178 (2008) 745 [arXiv:0710.2067] [InSPIRE].

[86] F. Mahmoudi, SuperIso v2.3: a program for calculating flavor physics observables in supersymmetry, Comput. Phys. Commun. 180 (2009) 1579 [arXiv:0808.3144] [INSPIRE].

[87] F. Mahmoudi, SuperIso v3.0, flavor physics observables calculations: extension to NMSSM, Comput. Phys. Commun. 180 (2009) 1718 [INSPIRE].

[88] HFLAV collaboration, Averages of b-hadron, c-hadron, and $\tau$-lepton properties as of 2018, Eur. Phys. J. C 81 (2021) 226 [arXiv:1909.12524] [INSPIRE].

[89] M. Misiak et al., Updated NNLO QCD predictions for the weak radiative B-meson decays, Phys. Rev. Lett. 114 (2015) 221801 [arXiv:1503.01789] [INSPIRE].

[90] W. Altmannshofer and P. Stangl, New physics in rare B decays after Moriond 2021, arXiv:2103.13370 [INSPIRE]. 
[91] ATLAS collaboration, Search for squarks and gluinos in final states with jets and missing transverse momentum using $139 \mathrm{fb}^{-1}$ of $\sqrt{\mathrm{s}}=13 \mathrm{TeV}$ pp collision data with the ATLAS detector, JHEP 02 (2021) 143 [arXiv: 2010.14293] [INSPIRE].

[92] CMS collaboration, Searches for physics beyond the standard model with the $M_{\mathrm{T} 2}$ variable in hadronic final states with and without disappearing tracks in proton-proton collisions at $\sqrt{s}=13 \mathrm{TeV}$, Eur. Phys. J. C 80 (2020) 3 [arXiv:1909.03460] [InSPIRE].

[93] ATLAS collaboration, Search for new phenomena with top quark pairs in final states with one lepton, jets, and missing transverse momentum in pp collisions at $\sqrt{s}=13 \mathrm{TeV}$ with the ATLAS detector, JHEP 04 (2021) 174 [arXiv:2012.03799] [INSPIRE].

[94] CMS collaboration, Search for top squark production in fully-hadronic final states in proton-proton collisions at $\sqrt{s}=13$ TeV, Phys. Rev. D 104 (2021) 052001 [arXiv:2103.01290] [INSPIRE].

[95] ATLAS collaboration, Search for electroweak production of charginos and sleptons decaying into final states with two leptons and missing transverse momentum in $\sqrt{s}=13 \mathrm{TeV} p p$ collisions using the ATLAS detector, Eur. Phys. J. C 80 (2020) 123 [arXiv:1908.08215] [INSPIRE].

[96] CMS collaboration, Search for electroweak production of charginos and neutralinos in proton-proton collisions at $\sqrt{s}=13 \mathrm{TeV}$, CMS-PAS-SUS-19-012 (2021).

[97] CMS collaboration, Search for supersymmetry in final states with two oppositely charged same-flavor leptons and missing transverse momentum in proton-proton collisions at $\sqrt{s}=13 \mathrm{TeV}$, JHEP 04 (2021) 123 [arXiv: 2012.08600] [INSPIRE].

[98] CMS collaboration, Search for direct pair production of supersymmetric partners to the $\tau$ lepton in proton-proton collisions at $\sqrt{s}=13$ TeV, Eur. Phys. J. C 80 (2020) 189 [arXiv: 1907.13179] [INSPIRE].

[99] ATLAS collaboration, Search for direct stau production in events with two hadronic $\tau$-leptons in $\sqrt{s}=13$ TeV pp collisions with the ATLAS detector, Phys. Rev. D 101 (2020) 032009 [arXiv: 1911.06660] [INSPIRE].

[100] ATLAS collaboration, Searches for electroweak production of supersymmetric particles with compressed mass spectra in $\sqrt{s}=13 \mathrm{TeV}$ pp collisions with the ATLAS detector, Phys. Rev. D 101 (2020) 052005 [arXiv:1911.12606] [INSPIRE].

[101] M. Endo, K. Hamaguchi and F. Takahashi, Moduli-induced gravitino problem, Phys. Rev. Lett. 96 (2006) 211301 [hep-ph/0602061] [INSPIRE].

[102] L. Covi, J.E. Kim and L. Roszkowski, Axinos as cold dark matter, Phys. Rev. Lett. 82 (1999) 4180 [hep-ph/9905212] [INSPIRE].

[103] S. Nakamura, K.-i. Okumura and M. Yamaguchi, Axionic mirage mediation, Phys. Rev. D 77 (2008) 115027 [arXiv:0803.3725] [INSPIRE].

[104] K. Choi, K.S. Jeong, W.-I. Park and C.S. Shin, Thermal inflation and baryogenesis in heavy gravitino scenario, JCAP 11 (2009) 018 [arXiv:0908.2154] [INSPIRE].

[105] A. Brandenburg, L. Covi, K. Hamaguchi, L. Roszkowski and F.D. Steffen, Signatures of axinos and gravitinos at colliders, Phys. Lett. B 617 (2005) 99 [hep-ph/0501287] [INSPIRE].

[106] ATLAS collaboration, Search for displaced leptons in $\sqrt{s}=13 \mathrm{TeV}$ pp collisions with the ATLAS detector, Phys. Rev. Lett. 127 (2021) 051802 [arXiv:2011.07812] [INSPIRE]. 
[107] OPAL collaboration, Search for stable and longlived massive charged particles in $e^{+} e^{-}$ collisions at $\sqrt{s}=130 \mathrm{GeV}$ to 209-GeV, Phys. Lett. B 572 (2003) 8 [hep-ex/0305031] [INSPIRE].

[108] CMS collaboration, Search for heavy stable charged particles with $12.9 \mathrm{fb}^{-1}$ of 2016 data, CMS-PAS-EXO-16-036, (2016).

[109] H. Baer, K.-Y. Choi, J.E. Kim and L. Roszkowski, Dark matter production in the early Universe: beyond the thermal WIMP paradigm, Phys. Rept. 555 (2015) 1 [arXiv: 1407.0017] [INSPIRE].

[110] H.K. Dreiner and V.M. Lozano, R-parity violation and direct stau pair production at the $L H C$, arXiv:2001.05000 [INSPIRE].

[111] K. Desch, S. Fleischmann, P. Wienemann, H.K. Dreiner and S. Grab, Stau as the lightest supersymmetric particle in R-parity violating SUSY models: discovery potential with early LHC data, Phys. Rev. D 83 (2011) 015013 [arXiv:1008.1580] [inSPIRE].

[112] D. Dercks, H. Dreiner, M.E. Krauss, T. Opferkuch and A. Reinert, R-parity violation at the LHC, Eur. Phys. J. C 77 (2017) 856 [arXiv: 1706. 09418] [InSPIRE].

[113] B.C. Allanach, A. Dedes and H.K. Dreiner, $R$ parity violating minimal supergravity model, Phys. Rev. D 69 (2004) 115002 [Erratum ibid. 72 (2005) 079902] [hep-ph/0309196] [INSPIRE].

[114] ATLAS collaboration, Search for supersymmetry in events with four or more charged leptons in $139 \mathrm{fb}^{-1}$ of $\sqrt{\mathrm{s}}=13 \mathrm{TeV}$ pp collisions with the ATLAS detector, arXiv:2103.11684 [INSPIRE].

[115] ATLAS collaboration, Search for trilepton resonances from chargino and neutralino pair production in $\sqrt{s}=13 \mathrm{TeV}$ pp collisions with the ATLAS detector, Phys. Rev. D 103 (2021) 112003 [arXiv: 2011.10543] [INSPIRE].

[116] L.L. Everett, I.-W. Kim, P. Ouyang and K.M. Zurek, Deflected mirage mediation: a framework for generalized supersymmetry breaking, Phys. Rev. Lett. 101 (2008) 101803 [arXiv:0804.0592] [INSPIRE].

[117] L.L. Everett, I.-W. Kim, P. Ouyang and K.M. Zurek, Moduli stabilization and supersymmetry breaking in deflected mirage mediation, JHEP 08 (2008) 102 [arXiv:0806.2330] [INSPIRE]. 